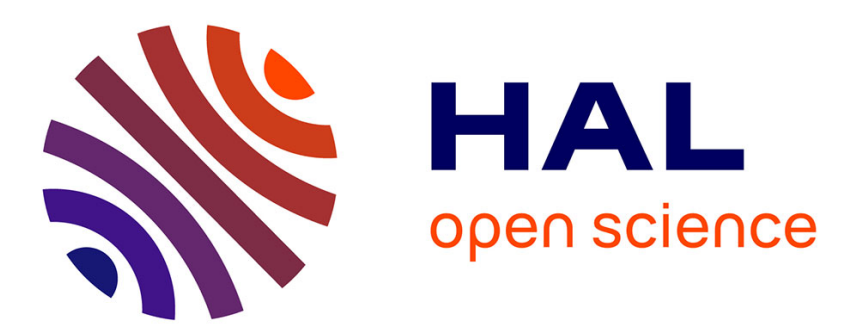

\title{
MedDRA® automated term groupings using OntoADR: evaluation with upper gastrointestinal bleedings
}

Julien Souvignet, Hadyl Asfari, Jérémy Lardon, Emilie del Tedesco, Gunnar

Declerck, Cédric Bousquet

\section{- To cite this version:}

Julien Souvignet, Hadyl Asfari, Jérémy Lardon, Emilie del Tedesco, Gunnar Declerck, et al.. MedDRA® automated term groupings using OntoADR: evaluation with upper gastrointestinal bleedings. Expert Opinion on Drug Safety, 2016, 15 (9), pp.1153 - 1161. 10.1080/14740338.2016.1206075 . hal01494025

\section{HAL Id: hal-01494025 \\ https://hal.sorbonne-universite.fr/hal-01494025}

Submitted on 22 Mar 2017

HAL is a multi-disciplinary open access archive for the deposit and dissemination of scientific research documents, whether they are published or not. The documents may come from teaching and research institutions in France or abroad, or from public or private research centers.
L'archive ouverte pluridisciplinaire HAL, est destinée au dépôt et à la diffusion de documents scientifiques de niveau recherche, publiés ou non, émanant des établissements d'enseignement et de recherche français ou étrangers, des laboratoires publics ou privés. 


\title{
MedDRA® automated term groupings using OntoADR: evaluation with upper gastrointestinal bleedings
}

Julien Souvignet, Hadyl Asfari, Jérémy Lardon, Emilie Del Tedesco, Gunnar Declerck \& Cédric Bousquet

\begin{abstract}
.
Objective: To propose a method to build customized sets of MedDRA ${ }^{1}$ terms when no appropriate grouping is available for the description of a medical condition. We illustrate this method with upper gastrointestinal bleedings (UGIB). In MedDRA, there is a dedicated SMQ for gastrointestinal hemorrhages but it does not allow users to distinguish between adverse drug reactions (ADRs) related to the upper or lower part of the digestive tract structure.

Research design and methods: We created a broad list of MedDRA terms related to UGIB and defined a gold standard with the help of experts. MedDRA terms were formally described in a semantic resource named OntoADR. We report the use of two semantic queries that automatically select candidate terms for UGIB. Query 1 is a combination of two SNOMED CT concepts describing both morphology "Hemorrhage" and finding site "Upper digestive tract structure". Query 2 complements Query 1 by taking into account additional MedDRA terms associated to two SNOMED CT concepts describing clinical manifestations "Melena" or "Hematemesis".
\end{abstract}

Results: We compared terms in queries and our gold standard achieving a recall of $71.0 \%$ and a precision of $81.4 \%$ for query 1 (F1 score 0.76 ); and a recall of $96.7 \%$ and a precision of $77.0 \%$ for query 2 (F1 score 0.86 ).

${ }^{1}$ MedDRA ${ }^{\circledR}$ is a registered trademark of the International Federation of Pharmaceutical Manufacturers and Associations. 
Conclusions: Our results demonstrate the feasibility of applying knowledge engineering techniques for building customized sets of MedDRA terms. Additional work is necessary on OntoADR to improve precision and recall and further evaluation on additional medical conditions should confirm the interest of the proposed strategy.

Keywords: Pharmacovigilance, MedDRA, SNOMED CT, Ontology, Signal detection, OntoADR, UGIB 


\section{Introduction}

Early identification of safety signals related to unknown adverse drug reactions (ADRs) and their confirmation is a key issue when treating patients [1]. Pre-marketing studies do not cover the heterogeneity of potential drug users (e.g. children or pregnant women) and usually lack statistical power to detect rare events due to limited number of patients and limited duration of clinical trials. Post-marketing drug surveillance, based on spontaneous reports, is a way to overcome these limitations.

Pharmacovigilance reports on alleged ADRs are typically coded with the MedDRA $^{\circledR 2}$ terminology [2,3] (Medical Dictionary for Drug Regulatory Activities). A critical issue, however, is that MedDRA allows high granularity and several terms may be used to code similar adverse events. Consequently, during a query in a pharmacovigilance database, a single code is not enough for targeting a medical condition; it is preferable to take into account a set of MedDRA codes that cover several aspects of a given condition.

Selection of ADR-related terms is difficult and time-consuming because terms can describe diseases, signs or symptoms, and even associated surgical procedures or abnormal investigation results. As terms related to a given condition can be found in different branches of MedDRA, the Maintenance and Support Services Organization (MSSO) has developed Standardized MedDRA Queries (SMQ) which are groupings of MedDRA terms constructed manually by expert consensus, that relate to a defined medical condition or area of interest and which are intended to support case identification 
[4]. SMQs do not solve every difficulty because they do not cover all medical conditions that may be related to a drug or may lack the required specificity $[5,6]$. Although one may search for "gastrointestinal hemorrhages" using the dedicated SMQ, there is no available sub-SMQ to distinguish between hemorrhages that are specific of the upper or lower part of the gastrointestinal tract. The MSSO invites MedDRA users to share their needs for new SMQs. But the delays for creating and publishing new SMQs are important. In addition, building new SMQs is a complex and costly expert-based process.

Our objective is to propose to MedDRA users an alternative method for building SMQ-like groupings by the means of an automated tool. We assume that knowledge engineering (methods and techniques from artificial intelligence that use knowledge to solve problems) may generate such groupings of MedDRA terms by enabling formal description (explicit and non-ambiguous definition) of these target medical conditions and logical computation thanks to a mechanism called terminological reasoning $[7,8]$ (mechanism allowing computers to reason logically using terminological knowledge). A formal definition is composed of logical assertions describing the relations between medical concepts. For example “ 'duodenum' is_part_of 'upper digestive tract structure' ” where 'duodenum' and 'upper digestive tract structure' are body parts, and 'is_part_of' is a semantic relation. We already described the construction of a semantic resource for ADRs, using description logic (DL), named OntoADR [9,10]. OntoADR contains MedDRA terms formally described with SNOMED CT concepts (Systematized

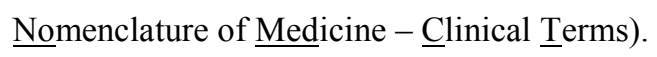

We describe in this paper how terminological reasoning performed on OntoADR resource may help MedDRA users to build new groupings of terms. We will apply our methodology and tools to an illustrative example based on upper gastrointestinal 
bleeding (UGIB). The evaluation consists of a comparison between a gold standard consisting of manually selected MedDRA terms, and our automatically generated group of OntoADR terms.

\section{Background}

\subsection{Upper gastrointestinal bleeding}

The upper gastrointestinal tract is defined as the portion of the digestive tract that is located from the esophagus to the ligament of Treitz [11]. UGIB is associated with significant morbidity and mortality [12]. The in-hospital mortality rate has been evaluated as $13 \%$ [13]. Increasing age and admission in hospital for co-morbidity, lead to significant higher mortality [14]. Minimizing morbidity and mortality requires early identification of these high-risk patients to allow appropriate intervention [15], and also risk factors such as drugs [16].

Several drugs may be associated with UGIB such as non-steroidal anti-inflammatory drugs (NSAIDs) [17], cyclo-oxygenase isoenzymes (COX) especially COX-2 inhibitors [18], corticosteroids [19], selective serotonin reuptake inhibitors (SSRIs) [20], antithrombotic medications (antiplatelet agents or anticoagulants) e.g. acetylsalicylic acid, clopidogrel, or warfarin [21], and spironolactone [22]. NSAIDs can damage the gastric mucosa by inhibiting COX-1 isoenzyme, and some may also have a direct effect due to their acidic property. Antithrombotic agents promote bleeding in the whole body such as SSRIs that inhibit the recapture of serotonin in platelets and may be responsible to impair the hemostatic function. 
Table 1 is a classification of causes of UGIB first by type of lesion e.g. ulcer or varice, and second by localization in the digestive tract from top to bottom. In some cases, blood originating from the intestine distal from the ligament of Treitz may travel to the upper part, e.g. intestinal diverticulum or angiodysplasia. We divided these causes in two categories related to major (e.g. ulcer) and minor causes of UGIB (e.g. hemobilia).

Table 1 - Causes of UGIB

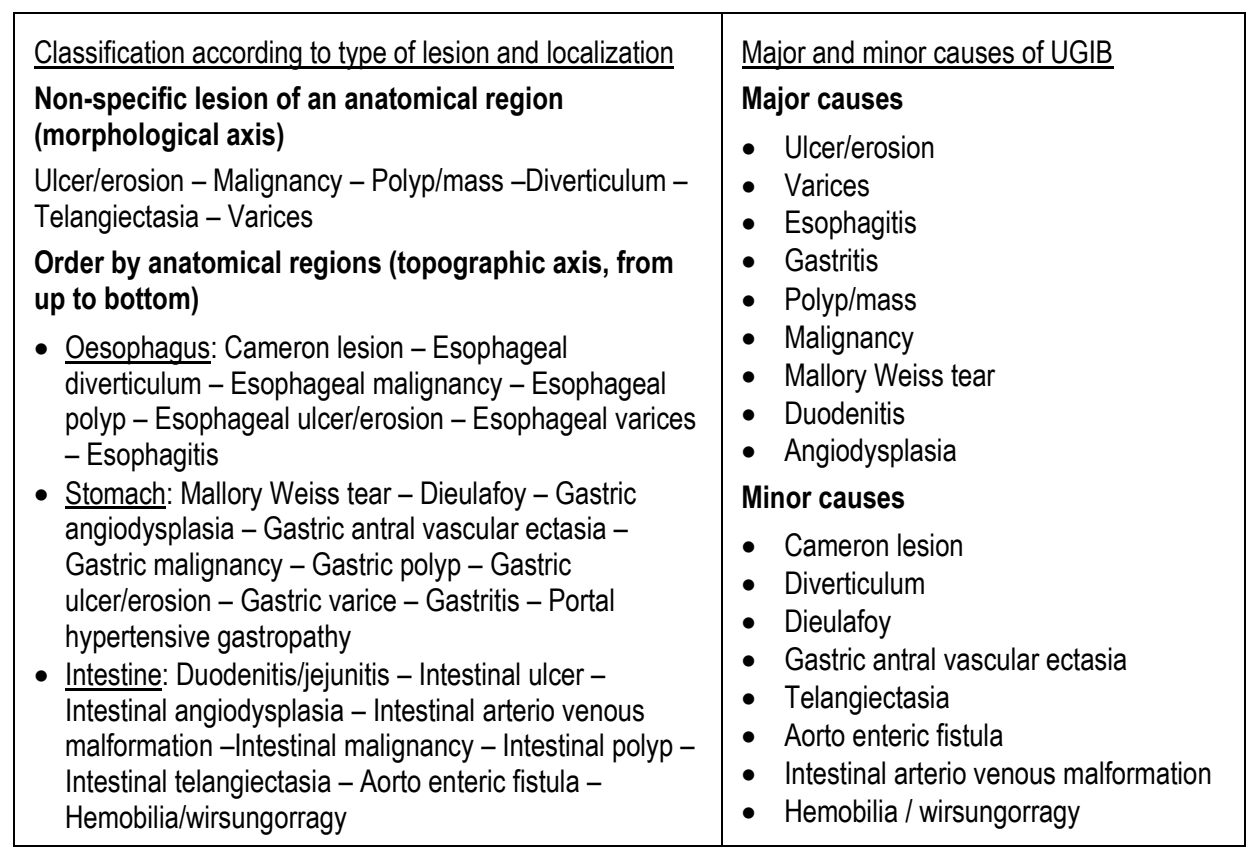

The most frequent signs and symptoms related to UGIB are hematemesis (vomiting bright red blood) and melena (black tarry stool) that occurs in $90 \%$ of digestive bleedings and is due to degradation of blood during gastrointestinal transit [11]. Whereas hematochezia (bloody or maroon-colored stool) may be a sign of massive UGIB, this test is usually dedicated to the screening of colon cancer [23] and prescription of such test is usually not indicative of UGIB. 


\subsection{SNOMED CT}

From the previous classification, it can be anticipated that modeling will take at least into account two important features (type of lesion and localization of this lesion), and will also involve other features such as abnormal body functions or severity which are not part of the MedDRA terminology. This is the reason why we relied on SNOMED CT, a terminology providing a broad coverage of clinical terms, including "body structure", "findings", “disorders", "substances" and "procedures", and other relevant categories.

SNOMED CT is, to our knowledge, the most complete and most detailed terminology of medicine which knowledge is available in formal way. SNOMED CT has been implemented in several countries to code medical problems in many electronic health records. We use it as a part of our knowledge base, especially to provide medical concepts absent from MedDRA such as body parts, morphologies (e.g. inflammation, hemorrhage, etc.), or manifestations (e.g. vomiting). Figure 1 shows an excerpt of the SNOMED CT hierarchy. Starting from the gastrointestinal tract structure, arrows represent a subsumption relation with subdivisions such as lower and upper gastrointestinal tract structure. The upper part consists of the esophagus, duodenum and stomach parts. 


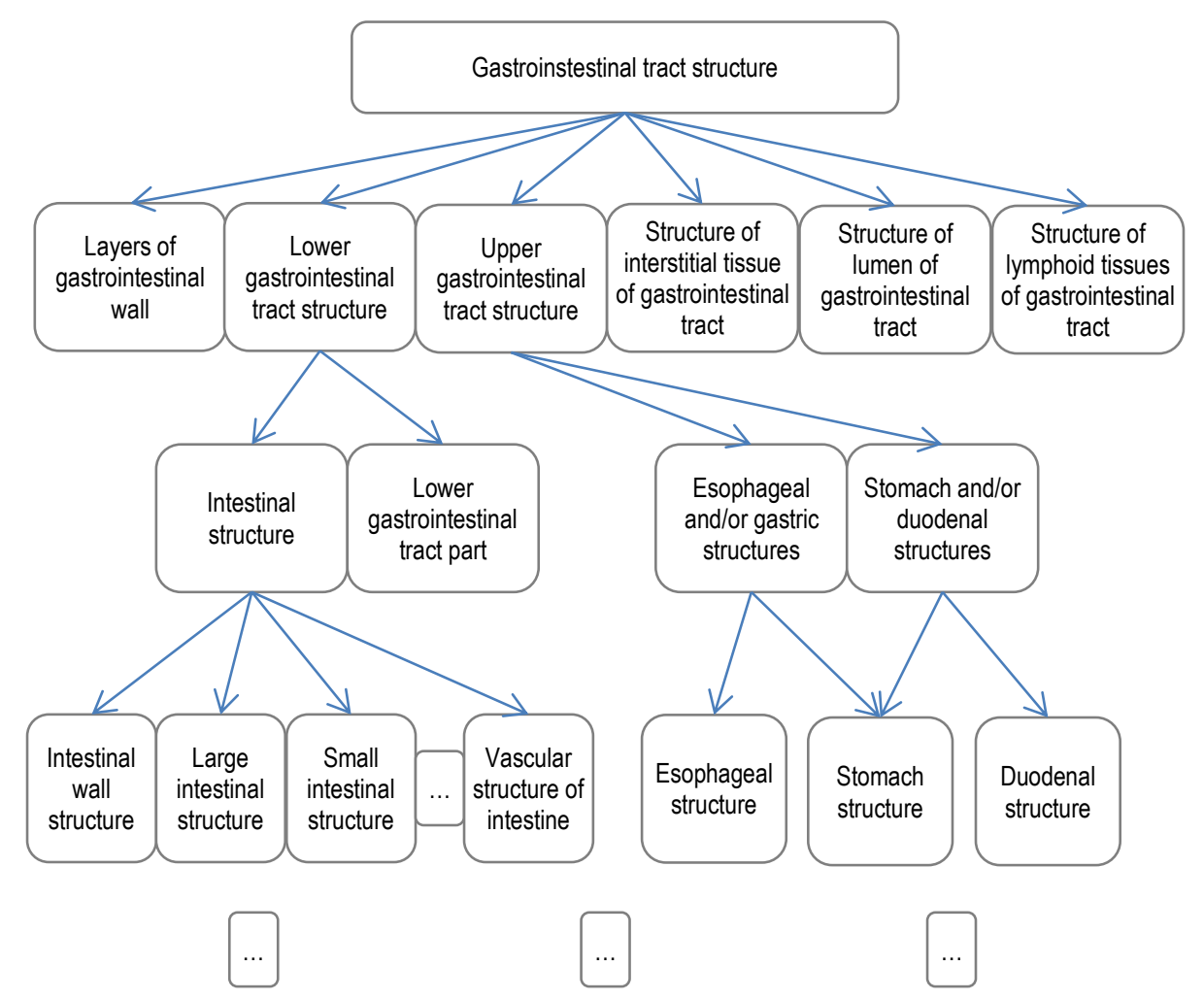

Figure 1 - Except of SNOMED CT Hierarchy of Gastrointestinal tract structure

\subsection{OntoADR}

OntoADR is a semantic resource using description logic that contains formal definitions of adverse drug reactions. Its purpose is to enable the use of terminological reasoning (i.e. automatic reasoning on the meaning of medical terms) for MedDRA terms. OntoADR includes 26 semantic properties inspired from SNOMED CT attributes, such as hasFindingSite, that describes the body part where the ADR is located, or hasAssociatedMorphology, that specifies the kind of morphologic abnormality one may 
observe e.g. a hemorrhage, a stenosis or an inflammation. A simplified representation of OntoADR is depicted in Figure 2.

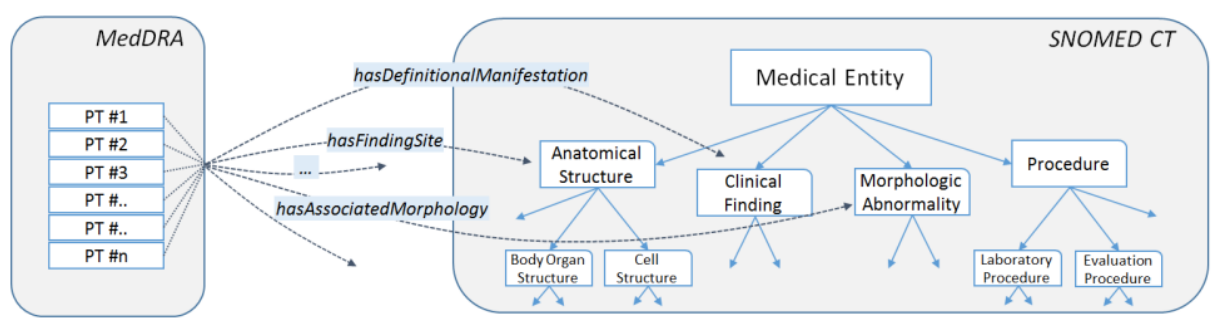

Figure 2 - OntoADR simplified representation

Today OntoADR has a semantic definition for $66.7 \%$ of MedDRA 17.0 terms. This means that these terms benefit from at least one semantic relation but we currently cannot guarantee these definitions are complete, and this explains why a curation by experts is required.

\section{4. $\mathrm{Ci} 4 \mathrm{SeR}$}

In order to maintain the OntoADR resource, we developed a tool named Ci4SeR (for Curation Interface for Semantic resources) [24]. It helps the processes of visualization, validation, edition and curation of MedDRA concepts. The curators are invited to validate definitions we were able to provide automatically or can modify the proposed properties or even add new ones.

For example, 'Gastroduodenitis haemorrhagic' is formally defined with several relations such as hasAssociatedMorphology 'Inflammation' OR 'Hemorrhage', and hasFindingSite 'Stomach structure' OR 'Duodenal structure'. This first set of relations defines necessary and sufficient conditions for the concept 'Gastroduodenitis 
hemorrhagic'. This means that the defined finding sites and morphological abnormalities will be present in every occurrence of such medical condition.

Curators added several properties that may be optional when describing a gastroduodenitis haemorrhagic condition. For example, the relations DueTo documents several potential causes of the disorder, e.g. 'portal hypertension' or 'non-steroidal antiinflammatory drugs'. The list of causal agents is not exhaustive and each relation is not part of necessary and sufficient conditions as one may observe occurrence of the disease that could be the consequence of different causal agents. The definition also provides some documentation on potential investigations such as Interprets 'Endoscopy' or signs and symptoms such as HasDefinitionalManifestation 'Nausea and vomiting'. The full definition of 'Gastroduodenitis haemorrhagic', as specified in $\mathrm{Ci} 4 \mathrm{SeR}$, is shown in Figure 3.

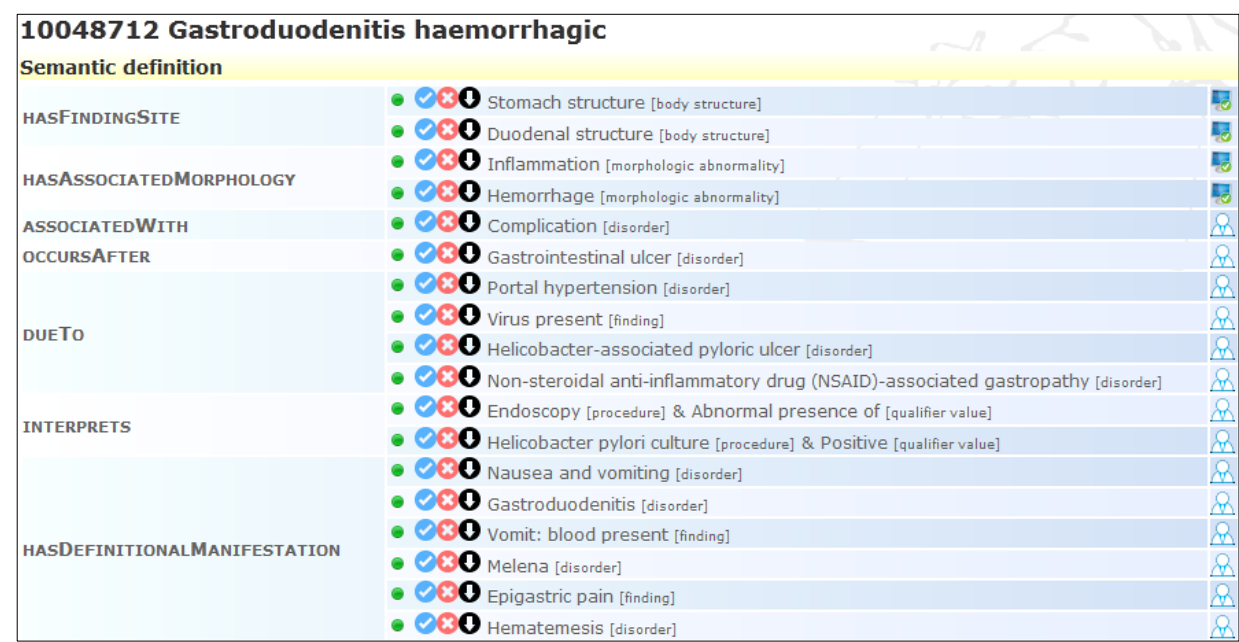

Figure 3 - Screenshot of the formal definition of the "Gastroduodenitis haemorrhagic" MedDRA term (id: 10048712) in Ci4SeR

Our automatic processes targeted the most used properties (mainly 'hasFindingSite' and 'hasAssociatedMorphology') and achieved a ratio of $62 \%$ properties that were 
provided automatically, while among other less used properties, this ratio dropped to $18 \%$ (due to a high number of manifestations and potential causes). For example, in this study, among the 31 MedDRA terms of our Gold Standard, we had 28 'hasFindingSite' properties provided automatically, 24 were validated and 4 removed. Curators also proposed 14 additional 'hasFindingSite' properties.

\subsection{OntoADR Query Tools}

The OntoADR Query Tools consist in a set of tools we developed to enable an easy, fast and user-friendly querying of the OntoADR semantic resource to find terms according to many criteria. It supports the creation of groupings of MedDRA terms on demand, based on multiple criteria. Querying benefits from logical reasoning such as subsumption between terms. For example, if one searches for terms defined as hasFindingSite 'Upper Digestive Tract Structure', one will get gastric haemorrhage among several terms because 'Upper Digestive Tract Structure' subsumes 'Stomach Structure'. The interface is still in development and will be the subject of a publication later.

\section{Materials and Methods}

\subsection{MedDRA terms}

In this study, we used MedDRA 17.0. This version contains 212 SMQs, including one named "Gastrointestinal hemorrhages" but a manual procedure is necessary to select those terms that describe medical conditions in the upper digestive tract. Therefore, UGIB illustrates an interesting example where MedDRA does not provide an existing 
grouping, and supporting term grouping thanks to a dedicated tool would be beneficial for the user who aims to query a pharmacovigilance database.

We created a broad reference grouping of terms about "Gastrointestinal bleedings" in order to include a wide range of MedDRA terms. We used hierarchical browsing and string search to select MedDRA terms. We first selected two reference groupings in MedDRA: the SMQ 'gastrointestinal haemorrhage' which contains 57 preferred terms (PT) and the high level term (HLT) 'Gastrointestinal haemorrhages' which contains 70 PT. We completed this list with a string search for terms matching *gastr*haemo* OR *gastr*bleed* in both lowest level term (LLT) and PT MedDRA labels, and we listed the 29 corresponding PT. The merging of these lists gave 91 distinct PTs.

This reference list of 91 terms has been blind-reviewed by 2 pharmacovigilance experts in order to extract only terms related to UGIB. When a disagreement occurred the two experts discussed the issue in order to reach a consensus. This list was then reviewed by a third expert, a gastroenterologist who checked the content and suggested the addition of several terms that were not initially selected. We considered this final list of 31 MedDRA terms as our gold standard. Lists of MedDRA terms and our gold standard are depicted as a Venn diagram in Figure 4 and excerpts are provided in Table 2 (the full lists are given as an additional content file). 


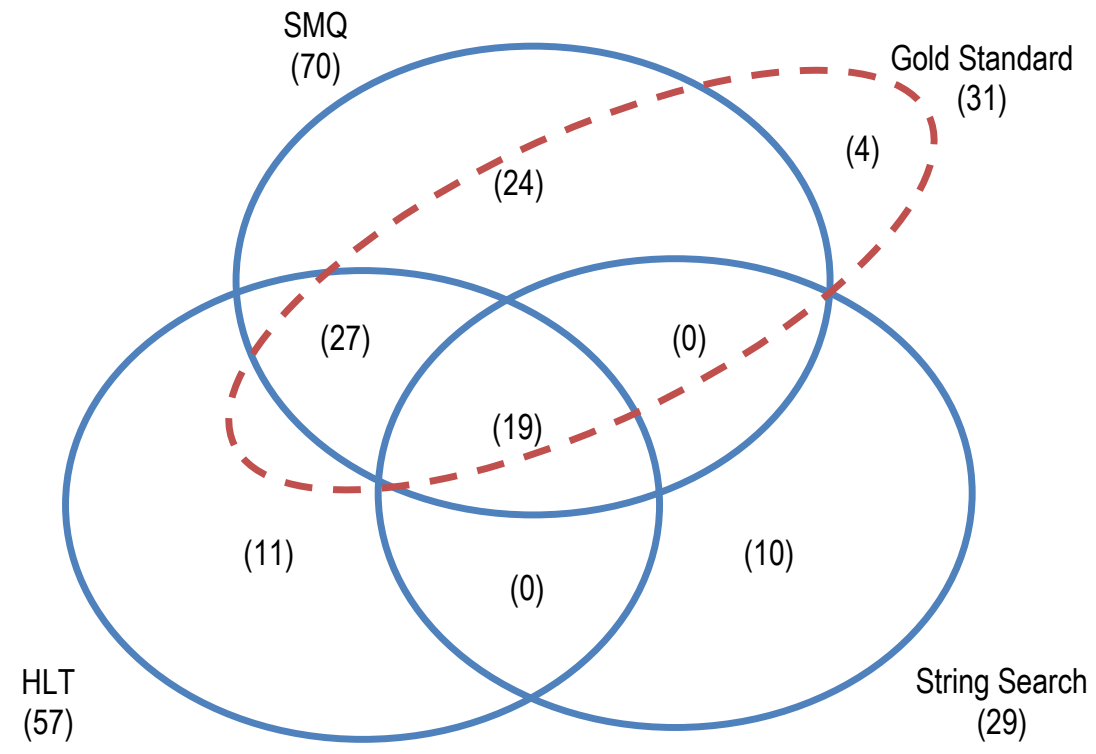

Figure 4- Venn diagram of the 3 MedDRA lists and our Gold Standard

Table 2 - Excerpt of MedDRA UGIB lists content

\begin{tabular}{|c|c|c|c|c|}
\hline $\begin{array}{c}\text { Common to the } 3 \\
\text { MedDRA lists }\end{array}$ & $\begin{array}{c}\text { Only in MedDRA } \\
\text { SMQ }\end{array}$ & $\begin{array}{c}\text { Only in MedDRA } \\
\text { HLT }\end{array}$ & $\begin{array}{l}\text { Only in MedDRA } \\
\text { string search }\end{array}$ & $\begin{array}{c}\text { Clinician-suggested } \\
\text { additions }\end{array}$ \\
\hline $\begin{array}{l}\text { Gastric } \\
\text { haemorrhage }\end{array}$ & $\begin{array}{l}\text { Abdominal wall } \\
\text { haematoma }\end{array}$ & $\begin{array}{l}\text { Anorectal varices } \\
\text { haemorrhage }\end{array}$ & $\begin{array}{l}\text { Anastomotic ulcer } \\
\text { perforation }\end{array}$ & $\begin{array}{l}\text { Dieulafoy's vascular } \\
\text { malformation }\end{array}$ \\
\hline $\begin{array}{l}\text { Gastritis } \\
\text { haemorrhagic }\end{array}$ & $\begin{array}{l}\text { Bloody peritoneal } \\
\text { effluent }\end{array}$ & $\begin{array}{l}\text { Duodenal vascular } \\
\text { ectasia }\end{array}$ & $\begin{array}{l}\text { Gastric ulcer } \\
\text { perforation }\end{array}$ & $\begin{array}{l}\text { Hemobilia } \\
\text { Angiodysplasia }\end{array}$ \\
\hline Gastroduodenal & Gingival bleeding & Gastric antral & Gastritis & Telangiectasia \\
\hline haemorrhage & Mouth haemorrhage & vascular ectasia & Gastritis alcoholic & \\
\hline $\begin{array}{l}\text { Gastrointestinal } \\
\text { haemorrhage }\end{array}$ & Pancreatic & $\begin{array}{l}\text { Gastric occult blood } \\
\text { positive }\end{array}$ & Gastritis atrophic & \\
\hline $\begin{array}{l}\text { Mallory-Weiss } \\
\text { syndrome }\end{array}$ & $\begin{array}{l}\text { Umbilical } \\
\text { haematoma }\end{array}$ & $\begin{array}{l}\text { Haemorrhoidal } \\
\text { haemorrhage }\end{array}$ & $\begin{array}{l}\text { oesophageal } \\
\text { variceal }\end{array}$ & \\
\hline $\begin{array}{l}\text { Upper } \\
\text { gastrointestinal } \\
\text { haemorrhage }\end{array}$ & $\ldots$ & $\begin{array}{l}\text { Ulcer haemorrhage } \\
\ldots\end{array}$ & $\begin{array}{l}\text { haemorrhage } \\
\text { prophylaxis } \\
\text {... }\end{array}$ & \\
\hline & & & & \\
\hline
\end{tabular}

\subsection{Semantic Queries}

In the current work, we used OntoADR (in version January 2015) which is based on MedDRA 17.0 version. We developed two queries in order to retrieve candidate terms 
for the UGIB medical condition. A first query was designed to identify any hemorrhage observable in the upper gastrointestinal tract.

hasFindingSite 'Upper gastrointestinal tract structure'

AND hasAssociatedMorphology 'Hemorrhage'

(Query 1)

Query 2 is targeting the actual manifestation of an UGIB, taking the risk to be broader than necessary.

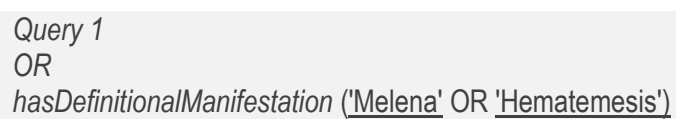

In order to complement findings using our gold standard, we also considered existing groupings in MedDRA that are usually selected as gold standards. First we reviewed the MedDRA 'gastrointestinal haemorrhage' SMQ, and selected only MedDRA terms that were related to bleeding in the upper part of the digestive system to design an SMQ-subset gold standard. Second, we used the 'Gastrointestinal haemorrhages' HLT to get an HLT-subset gold standard.

The results of each query were evaluated using measures of precision, recall and F1 measure. We also evaluated the number of additional case reports that would be selected in the Adverse Events Reporting System (AERS) of the Food and Drug Administration (FDA) if terms resulting from our semantic queries but absent from the gold standard were employed for searching case reports.

\section{Results}

Our gold standard and the terms obtained for the queries can be viewed in Figure 5. In Query 1 we observed that 9 preferred terms present in our Gold Standard were absent:

$$
\text { Query } 1 \text { Recall }=\frac{\text { relevant terms } \cap \text { retrieved terms }}{\text { total relevant terms }}=71.0 \%
$$




$$
\begin{gathered}
\text { Query } 1 \text { Precision }=\frac{\text { relevant terms } \cap \text { retrieved terms }}{\text { retrieved terms }}=81.4 \% \\
\text { Query } 1 F 1 \text { Score }=2 \frac{\text { Precision } * \text { Recall }}{\text { Precision }+ \text { Recall }}=0.758
\end{gathered}
$$

But 8 of them (such as 'Melaena' or 'Occult blood positive') where identified in Query 2:

$$
\begin{gathered}
\text { Query } 2 \text { Recall }=\frac{\text { relevant terms } \cap \text { retrieved terms }}{\text { total relevant terms }}=96.7 \% \\
\text { Query } 2 \text { Precision }=\frac{\text { relevant terms } \cap \text { retrieved terms }}{\text { retrieved terms }}=77.0 \% \\
\text { Query } 2 \text { F1 Score }=2 \frac{\text { Precision } * \text { Recall }}{\text { Precision }+ \text { Recall }}=0.857
\end{gathered}
$$




\begin{tabular}{|c|c|c|c|c|c|c|c|c|}
\hline \multicolumn{2}{|c|}{ Upper Gastro-Intestinal Bleedings } & & & & & & & \\
\hline \multicolumn{6}{|l|}{ Gold Standard } & \multicolumn{3}{|l|}{ OntoADR Query 2} \\
\hline MedDRA term & SMQ & HLT & Txt Search & Query 1 & Query 2 & MedDRA term & Query 1 & Gold Std \\
\hline Angiodysplasia & No & No & No & No & Yes & Anastomotic ulcer haemorrhage & Yes & No \\
\hline Chronic gastrointestinal bleeding & Yes & Yes & Yes & Yes & Yes & Angiodysplasia & No & Yes \\
\hline Dieulafoy's vascular malformation & No & No & No & No & Yes & Aorto-oesophageal fistula & Yes & No \\
\hline Duodenal ulcer haemorrhage & Yes & Yes & No & Yes & Yes & Chronic gastrointestinal bleeding & Yes & Yes \\
\hline Duodenitis haemorrhagic & Yes & Yes & No & Yes & Yes & Dieulafoy's vascular malformation & No & Yes \\
\hline Gastric antral vascular ectasia & No & Yes & No & Yes & Yes & Duodenal ulcer haemorrhage & Yes & Yes \\
\hline Gastric haemorrhage & Yes & Yes & Yes & Yes & Yes & Duodenal ulcer perforation & No & No \\
\hline Gastric ulcer haemorrhage & Yes & Yes & Yes & Yes & Yes & Duodenal varices & Yes & No \\
\hline Gastric ulcer haemorrhage, obstructive & Yes & Yes & Yes & Yes & Yes & Duodenitis haemorrhagic & Yes & Yes \\
\hline Gastric varices haemorrhage & Yes & Yes & Yes & Yes & Yes & Erosive duodenitis & Yes & No \\
\hline Gastritis alcoholic haemorrhagic & Yes & Yes & Yes & Yes & Yes & Gastric antral vascular ectasia & Yes & Yes \\
\hline Gastritis haemorrhagic & Yes & Yes & Yes & Yes & Yes & Gastric haemorrhage & Yes & Yes \\
\hline Gastroduodenal haemorrhage & Yes & Yes & Yes & Yes & Yes & Gastric ulcer haemorrhage & Yes & Yes \\
\hline Gastroduodenitis haemorrhagic & Yes & Yes & Yes & Yes & Yes & Gastric ulcer haemorrhage, obstructive & Yes & Yes \\
\hline Gastrointestinal haemorrhage & Yes & Yes & Yes & No & Yes & Gastric ulcer perforation & No & No \\
\hline Gastrointestinal ulcer haemorrhage & Yes & Yes & Yes & No & Yes & Gastric varices haemorrhage & Yes & Yes \\
\hline Haematemesis & Yes & Yes & No & Yes & Yes & Gastritis alcoholic haemorrhagic & Yes & Yes \\
\hline Haemobilia & No & No & No & Yes & Yes & Gastritis haemorrhagic & Yes & Yes \\
\hline Haemorrhagic erosive gastritis & Yes & Yes & No & Yes & Yes & Gastroduodenal haemorrhage & Yes & Yes \\
\hline Mallory-Weiss syndrome & Yes & Yes & Yes & Yes & Yes & Gastroduodenitis haemorrhagic & Yes & Yes \\
\hline Melaena & Yes & Yes & No & No & Yes & Gastrointestinal haemorrhage & No & Yes \\
\hline Melaena neonatal & Yes & Yes & No & No & Yes & Gastrointestinal ulcer haemorrhage & No & Yes \\
\hline Neonatal gastrointestinal haemorrhage & Yes & Yes & Yes & No & Yes & Haematemesis & Yes & Yes \\
\hline Occult blood positive & No & Yes & No & No & Yes & Haemobilia & Yes & Yes \\
\hline Oesophageal haemorrhage & Yes & Yes & No & Yes & Yes & Haemorrhagic erosive gastritis & Yes & Yes \\
\hline Oesophageal ulcer haemorrhage & Yes & Yes & No & Yes & Yes & Intestinal haemorrhage & No & No \\
\hline Oesophageal varices haemorrhage & Yes & Yes & Yes & Yes & Yes & Mallory-Weiss syndrome & Yes & Yes \\
\hline Oesophagitis haemorrhagic & Yes & Yes & No & Yes & Yes & Melaena & No & Yes \\
\hline Peptic ulcer haemorrhage & Yes & Yes & No & Yes & Yes & Melaena neonatal & No & Yes \\
\hline Telangiectasia & No & No & No & No & No & Occult blood positive & No & Yes \\
\hline Upper gastrointestinal haemorrhage & Yes & Yes & Yes & Yes & Yes & Oesophageal haemorrhage & Yes & Yes \\
\hline $\operatorname{TOTAL}(/ \mathbf{3 1})$ & $25(81 \%)$ & $27(87 \%)$ & $15(48 \%)$ & $22(71 \%)$ & $30(97 \%)$ & Oesophageal ulcer haemorrhage & Yes & Yes \\
\hline & & & & & & Oesophageal varices haemorrhage & Yes & Yes \\
\hline & & & & & & Oesophagitis haemorrhagic & Yes & Yes \\
\hline & & & & & & Neonatal gastrointestinal haemorrhage & No & Yes \\
\hline & & & & & & Peptic ulcer haemorrhage & Yes & Yes \\
\hline & & & & & & Peptic ulcer perforation & Yes & No \\
\hline & & & & & & Portal hypertensive gastropathy & No & No \\
\hline & & & & & & Upper gastrointestinal haemorrhage & Yes & Yes \\
\hline & & & & & & TOTAL (/39) & $27(69 \%)$ & $29(77 \%)$ \\
\hline
\end{tabular}

Figure 5 - Gold standard vs MedDRA groupings vs OntoADR grouping results

Only one term is absent from Query 2: 'Telangiectasia', but 10 additional PT were retrieved by our queries (such as 'Aorto-oesophageal fistula', 'Erosive duodenitis', or 'Portal hypertensive gastropathy').

Results were very close with the SMQ-subset gold standard (query 1: recall 80.0\%, precision $81.4 \%$; query 2 : recall $100 \%$, precision $65.8 \%$ ) and similarly with the HLTsubset (query 1: recall $81.5 \%$, precision $81.5 \%$; query 2: recall 100\%, precision $69.2 \%$ ).

The 10 additional terms retrieved in query 2 but absent from the gold standard could lead to $10 \%$ more cases when searching UGIB reports in the AERS pharmacovigilance database. 


\section{Discussion}

\subsection{UGIB Terms}

We selected the UGIB safety topic because it was a good example of the difficulty when selecting terms in MedDRA in order to build custom groupings. This difficulty is especially sensible when no predefined groupings are available. Even if such a grouping exists, it would only represent the view of the experts that designed it. Indeed, MedDRA existing groupings (SMQ 'gastrointestinal haemorrhage' and the HLT 'Gastrointestinal haemorrhages') are discordant (see 2.2). Furthermore, the SMQ "gastrointestinal haemorrhage", since its introduction, has been modified 7 times. Overall, 12 terms have been added by experts $(+24 \%)$ while rules for inclusion and exclusion remained unchanged.

We then chose to create a new gold standard and decided to take a broad approach by considering all medical conditions related to UGIB.

Our expert selected 'Telangiectasia', that are small dilated blood vessels, not specific to UGIB. The term 'Gastrointestinal telangiectasia' was not available in MedDRA 17.0 and has since been introduced in 18.0. Choosing 'Telangiectasia' may relate conditions of telangiectasia that are located in multiple parts of the body especially on the skin. In OntoADR, this term is defined as located in the skin and mucous membrane structure, and not the gastrointestinal structure, so it was not retrieved by our query.

For additional terms, 'Erosive duodenitis' may be related to an inflammation of the duodenal wall as part of a drug action but may not be necessarily associated with a hemorrhage. Other etiologies than a drug should be considered for the remaining terms. 
For example, a portal hypertension may cause changes in the mucosa of the stomach and may induce 'Portal hypertensive gastropathy'. Blood may be originating from other parts of the body than the digestive tract such as in the case of a pathology of aorta where the blood flows from the aorta to esophagus in patients presenting 'Aorto-esophageal fistula'.

We observed in a quantitative way that additional terms retrieved in query 2 but absent from the gold standard would eventually allow to detect more UGIB case reports in a pharmacovigilance database. However, these additional terms may also lead to false positives, which explains why a secondary analysis would be useful in order to assess the potential impact of using these terms when searching in a database. Indeed, it is not sufficient to have the relevant MedDRA terms when searching in a pharmacovigilance database to get all relevant case reports, as demonstrated by Géniaux et al, who observed heterogeneous performance of SMQs for case retrieval [25]. We plan to investigate this issue in future work, following an approach comparable to the former authors' protocole, in order to figure the actual impact of using different terms to retrieve case reports.

\subsection{Related work}

MedDRA terms groupings to detect UGIB have already been built manually in the past, e.g. in the EU-ADR project [26] and reused in the Safety of Non-steroidal Antiinflammatory Drugs (SOS) European project [27]. Whereas the MedDRA SMQ does not contain the 'Melena' and 'Hematemesis' PT, such terms were selected in studies focusing on UGIB $[26,28]$ and we expect that addition of such terms might be desirable. But, contrary to these studies we chose to exclude from the UGIB grouping terms describing perforations (e.g. 'Gastric perforation' [26]) or digestive ulcers (e.g. 'peptic ulcer' [28]) which are not specified as bleeding in MedDRA. Indeed one may observe 
gastric ulcers or gastric perforation that might not be associated with bleeding. In these studies, selection of terms was performed manually without a dedicated query tool and may benefit from the approach we present here.

Other ontologies have been described for adverse events: OAE (Ontology of adverse events) [29] and AERO (Adverse event reporting ontology) [30], that may be useful in the field of pharmacovigilance. AERO proposes an alternative approach to the identification of case reports describing anaphylaxis using the Automatic Brighton Classification (ABC) tool [31]; its scope is therefore limited and MedDRA terms are not formally described according to relations we propose such as finding site, abnormal morphology. OAE contains about 1900 terms describing ADRs that are mapped to the corresponding MedDRA terms. Therefore, all MedDRA terms potentially coded in a pharmacovigilance database might not be cross referenced. Additionally, in OAE, ADRs are classified as descendants of pathological bodily process which does not allow investigation results or procedures to qualify as candidate terms for a SMQ, whereas they may be coded in case reports (e.g. 'Creatinine increased' or 'Dialysis' may be indicative of a potential 'Renal insufficiency').

\subsection{Formalization of MedDRA}

Some authors have recently proposed to use formal semantics to improve traditional statistical-based methods for detecting signals in pharmacovigilance [32]. The new method we propose for grouping MedDRA terms may help to achieve this goal. The tools and methods presented in this article are, however, still experimental. Several actions are required in order to evolve towards an operational version that may be used in daily routine by pharmacovigilance teams. First, before the functionalities enabled by 
OntoADR can be implemented in future versions of MedDRA, there is a need to discuss with MedDRA users (pharmacovigilance departments within drug regulatory agencies or the pharmaceutical industry), and stakeholders such as the MSSO or the ICH (International Conference on Harmonization). Second, there is a need to complete and evaluate current formal definitions to ensure that they correctly take into account most MedDRA terms currently coded in pharmacovigilance databases. Third, studies must be conducted to evaluate the usability of the OntoADR Query tools, and whether it is appropriate to users' needs and work methods.

\subsection{Semi-automatic groupings}

We previously described preliminary findings of the current methodology in 2012, at the First workshop on computational methods in pharmacovigilance [33]. The current work improves the proposed methodology in several ways. The OntoADR resource has also been improved thanks to the curation process and now benefits from new functionalities such as the possibility to exclude criteria or the calculation of common properties between multiple terms.

Performing queries on the OntoADR formal definitions requires skills in knowledge engineering that are lacking for most pharmacovigilance experts. For this reason, we developed the OntoADR Query Tools that provide a user-friendly interface to execute such queries without the difficulty to learn complex theories on redaction of semantic queries in specific languages such as SPARQL [34] or in the Manchester Syntax language using an ontology editing software. However, facilitating queries on the user's side is dependent from an important workload on the knowledge engineer's side who has to implement formal definitions of MedDRA terms in a rigorous way. 
We previously tested our algorithm on a preliminary version of OntoADR and a more restricted list of UGIB terms and observed slightly better performances [33]. While we considered in the first version bleeding that was originating from the upper digestive tract, we took into account additional pathologies where blood is produced from another part of the body but may flow to the upper digestive tract, e.g. 'hemobilia' or 'angiodysplasia'. As these terms are not formally defined with a potential finding site in the upper digestive tract, thus they were not always retrieved by our query, impacting precision and recall.

We conducted several formal studies, demonstrating that the proposed approach may be generalized to a broader set of medical conditions. We used Trifirò et al. [35] ranked list of 23 "safety topics" (top importance pharmacovigilance events to monitor) which included 'cutaneous bullous eruptions', 'acute renal failure' or 'upper gastrointestinal bleeding' (UGIB). E.g., in a previous study, we applied the same methodology on bullous eruptions reaching recall of $100 \%$ and precision of $78.6 \%$ by identifying six additional terms that were absent from the SMQ such as 'Oropharyngeal blistering' or 'Oral mucosal blistering' [36]. In another study, we were also able to identify the MedDRA terms on anaphylaxis. We reached a precision and recall of $100 \%$ for our grouping vs. the corresponding HLT, and $71 \%$ recall and $71 \%$ precision versus Anaphylaxis SMQ, identifying two additional terms: 'Anaphylactoid syndrome of pregnancy' and 'First use syndrome' [37]. The second term has since been integrated into the SMQ in an updated version of MedDRA.

In a future version of OntoADR, we plan to separate necessary and sufficient conditions from other relations that may be true only in some occurrences of the disease using the 'may' prefix, e.g. MayHaveDefinitionalManifestation 'Epigastric pain'. 
We now plan to make such analysis on the full Trifirò's list of 23 safety topics, in order to demonstrate that our approach brings strong and sustainable added value to MedDRA.

\section{Conclusion}

This article shows the difficulty of creating MedDRA groups of terms for a specific condition but depicts the advantages of our approach based on terminological reasoning. The results described in this article demonstrate that our knowledge-based semiautomatic method and associated set of tools for selecting and grouping MedDRA terms can efficiently support the realization of ADR groupings. Our approach allows to design different groupings for a given safety topic by taking into account the preferred strategy of the expert rather than relying on fixed groupings.

Our method offers high levels of recall. It is more efficient for an expert to review and filter relevant terms in a complete list rather than executing and merging multiple queries with the risk of not being exhaustive. This explains why favoring recall to precision is a preferable strategy.

The proposed approach may provide further flexibility and support to drug safety experts in retrieving, selecting and analyzing individual case reports and also perform signal detection in spontaneous reports databases.

\section{Acknowledgments}

The research leading to these results was conducted as part of the PROTECT consortium (Pharmacoepidemiological Research on Outcomes of Therapeutics by a European ConsorTium, www.imi-protect.eu) which is a public-private partnership 
coordinated by the European Medicines Agency. The PROTECT project has received support from the Innovative Medicine Initiative Joint Undertaking (www.imi.europa.eu) under Grant Agreement $\mathrm{n}^{\circ} 115004$, resources of which are composed of financial contribution from the European Union's Seventh Framework Programme (FP7/20072013) and EFPIA companies' in kind contribution.

We acknowledge Eric Sadou, Adrien Fanet and Anne Jamet who contributed to the development of OntoADR.

The views expressed are those of the authors only. The authors have no conflict of interest with the subject matter discussed in the manuscript.

\section{References}

1. Meyboom RHB, Egberts ACG, Edwards IR, Hekster YA, de Koning FHP, Gribnau FWJ. Principles of signal detection in pharmacovigilance. Drug Saf1997;16(6):355-65.

2. Mozzicato P. MedDRA: an overview of the medical dictionary for regulatory activities. Pharmaceut Med $2009 ; 23: 65-75$.

3. Introductory Guide MedDRA, MSSO, 2012, http://meddramsso.com/files_acrobat/intguide_15_0_ English_update.pdf

4. Mozzicato P. Standardised MedDRA queries: their role in signal detection. Drug Saf 2007:30(7): 617-9.

5. Pearson RK, Hauben M, Goldsmith DI, Gould AL, Madigan D, O’Hara DJ, Reisinger SJ, Hochberg AM. Influence of the MedDRA hierarchy on pharmacovigilance data mining results. Int $\mathrm{J}$ Med Inform 2009;78(12):97-103.

6. Hill R, Hopstadius J, Lerch M, Noren N. An attempt to expedite signal detection by grouping related adverse reaction terms. Drug Saf 2012;35(12): 1194-5. 
7. Bousquet C, Lagier G, Lillo-Le Louët A, Le Beller C, Venot A, Jaulent MC. Appraisal of the MedDRA conceptual structure for describing and grouping adverse drug reactions. Drug Saf. 2005;28(1):19-34.

8. Henegar C, Bousquet C, Lillo-Le Louët A, Degoulet P, Jaulent MC. Building an ontology of adverse drug reactions for automated signal generation in pharmacovigilance. Comput Biol Med. 2006;36(78):748-67.

9. Bousquet C, Sadou É, Souvignet J, Jaulent MC, Declerck G. Formalizing MedDRA to support semantic reasoning on adverse drug reaction terms. J Biomed Inform 2014;49:282-91.

10. Declerck G, Bousquet C, Jaulent MC. Automatic generation of MedDRA terms groupings using an ontology. StudHealth Technol Inform 2012;180:73-7.

11. Cappell MS, Friedel D. Initial management of acute upper gastrointestinal bleeding: from initial evaluation up to gastrointestinal endoscopy. Med Clin North Am 2008;92(3):491-509.

12. Wilkins T, Khan N, Nabh A, Schade RR. Diagnosis and management of upper gastrointestinal bleeding. Am Fam Physician 2012;85(5):469-76.

13. Van Leerdam ME, Vreeburg EM, Rauws EA, Geraedts AA, Tijssen JG, Reitsma JB, Tytgat GN. Acute upper GI bleeding: did anything change? Time trend analysis of incidence and outcome of acute upper GI bleeding between 1993/1994 and 2000. Am J Gastroenterol 2003;98(7):1494-9.

14. Van Leerdam ME. Epidemiology of acute upper gastrointestinal bleeding. Best Pract Res ClinGastroenterol 2008;22(2):209-24.

15. Barkun AN, Bardou M, Kuipers EJ, Sung J, Hunt RH, Martel M, Sinclair P; International Consensus Upper Gastrointestinal Bleeding Conference Group. International consensus recommendations on the management of patients with nonvariceal upper gastrointestinal bleeding. Ann Intern Med 2010;152(2):101-13

16. Masclee GM, Valkhoff VE, Coloma PM, de Ridder M, Romio S, Schuemie MJ, Herings R, Gini R, Mazzaglia G, Picelli G, Scotti L, Pedersen L, Kuipers EJ, van der Lei J, Sturkenboom MC. Risk of upper gastrointestinal bleeding from different drug combinations. Gastroenterology. 2014;147(4):784-792.e9.

17. Massó González EL, Patrignani P, Tacconelli S, García Rodríguez LA. Variability among non-steroidal anti-inflammatory drugs in risk of upper gastrointestinal bleeding. Arthritis Rheum 2010;62(6):1592-601. 
18. Nørgård B, Pedersen L, Johnsen SP, Tarone RE, McLaughlin JK, Friis S, Sørensen HT. COX-2-selective inhibitors and the risk of upper gastrointestinal bleeding in high-risk patients with previous gastrointestinal diseases: a population-based case-control study. Aliment Pharmacol Ther. 2004;19(7):817-25.

19. Hernández-Díaz S, Rodríguez LA. Steroids and risk of upper gastrointestinal complications. Am J Epidemiol. 2001;153(11):1089-1093.

20. De Abajo FJ, Montero D, Rodríguez LA, Madurga M. Antidepressants and risk of upper gastrointestinal bleeding. Basic ClinPharmacolToxicol 2006;98(3):304-10.

21. Delaney JA, Opatrny L, Brophy JM, Suissa S. Drug drug interactions between antithrombotic medications and the risk of gastrointestinal bleeding. CMAJ 2007;177(4):347-51.

22. Verhamme K, Mosis G, Dieleman J, Stricker B, Sturkenboom M. Spironolactone and risk of upper gastrointestinal events: population based case-control study. BMJ 2006;333(7563):330.

23. Levin B, Lieberman DA, McFarland B et al. Screening and surveillance for the early detection of colorectal cancer and adenomatous polyps, 2008: a joint guideline from the American Cancer Society, the US Multi-Society Task Force on Colorectal Cancer, and the American College of Radiology. CA Cancer J Clin 2008;58(3):130-60.

24. Souvignet J, Asfari H, Declerck G, Lardon J, Trombert-Paviot B, Jaulent MC, Bousquet C.Ci4SeR curation interface for semantic resources - evaluation with adverse drug reactions. Stud Health Technol Inform. 2014;205:116-20.

25. Géniaux H, Assaf D, Miremont-Salamé G, Raspaud B, Gouverneur A, Robinson P, Pariente A, Salvo F. Performance of the standardised MedDRA ${ }^{\circledR}$ queries for case retrieval in the French spontaneous reporting database. Drug Saf 2014 Jul;37(7):537-42.

26. Avillach P, Mougin F, Joubert M et al. A semantic approach for the homogeneous identification of events in eight patient databases: a contribution to the European eu-ADR project. Stud Health Technol Inform. 2009;150:190-4.

27. Salvo F, Fourrier-Réglat A, Bazin F, et al. Cardiovascular and gastrointestinal safety of NSAIDs: a systematic review of meta-analyses of randomized clinical trials. Clin Pharmacol Ther 2011;89(6):85566. 
28. De Abajo FJ, Gil MJ, Bryant V, Timoner J, Oliva B, García-Rodríguez LA. Upper gastrointestinal bleeding associated with NSAIDs, other drugs and interactions: a nested case-control study in a new general practice database. Eur J ClinPharmacol 2013;69(3):691-701.

29. He Y, Sarntivijai S, Lin Y, Xiang Z, Guo A, Zhang S, Jagannathan D, Toldo L, Tao C, Smith B. OAE: the ontology of adverse events. J Biomed Semantics 2014;5:29.

30. Courtot M, Brinkman RR, Ruttenberg A. The logic of surveillance guidelines: an analysis of vaccine adverse event reports from an ontological perspective. PLoS One. 2014;9(3):e92632..

31. Botsis T, Woo EJ, Ball R. Application of information retrieval approaches to case classification in the vaccine adverse event reporting system. Drug Saf 2013;36(7):573-82

32. Koutkias VG, Jaulent MC. Computational approaches for pharmacovigilance signal detection: toward integrated and semantically-enriched frameworks. Drug Saf 2015;38(3):219-32.

33. Souvignet J, Declerck G, Jaulent MC, Bousquet C. Evaluation of Automated Term Groupings for Detecting Upper Gastrointestinal Bleeding Signals for Drugs, Drug Saf 2012;35(12):1195-6.

34. Harris S, Seaborne A, Prud'hommeaux E. SPARQL 1.1 Query Language (2013). W3C Recommendation, 2014.

35. Trifirò G, Pariente A, Coloma PM et al. Data mining on electronic health record databases for signal detection in pharmacovigilance: which events to monitor? Pharmacoepidemiol Drug Saf 2009;18(12):1176-84.

36. Asfari H, Souvignet J, Guy C, Bousquet C. Knowledge-based method for automated generation of new MedDRA Grouping for bullous conditions. Fundam Clin Pharmacol 2014;28 (suppl. 1):101

37. Souvignet J, Declerck G, Trombert B, Rodrigues JM, Jaulent MC, Bousquet C.. Evaluation of automated term groupings for detecting anaphylactic shock signals for drugs. AMIA Annu Symp Proc 2012;2012:882-90. 